\title{
The spectral excess theorem for graphs with few eigenvalues whose distance-2 or distance-1-or-2 graph is strongly regular *
}

\author{
C. Dalfó ${ }^{a}$, M.A. Fiol ${ }^{a, b}$, J. Koolen $^{c}$ \\ ${ }^{a}$ Universitat Politècnica de Catalunya, BarcelonaTech \\ Dept. de Matemàtiques, Barcelona, Catalonia \\ ${ }^{b}$ Barcelona Graduate School of Mathematics \\ \{cristina.dalfo,miguel.angel.fiol\}@upc.edu \\ ${ }^{c}$ University of Science and Technology of China \\ School of Mathematical Sciences \\ Hefei, Anhui, China \\ koolen@ustc.edu.cn
}

口

\begin{abstract}
We study regular graphs whose distance-2 graph or distance-1-or-2 graph is strongly regular. We provide a characterization of such graphs $\Gamma$ (among regular graphs with few distinct eigenvalues) in terms of the spectrum and the mean number of vertices at maximal distance $d$ from every vertex, where $d+1$ is the number of different eigenvalues of $\Gamma$. This can be seen as another version of the so-called spectral excess theorem, which characterizes in a similar way those regular graphs that are distance-regular.
\end{abstract}

Keywords: Distance-regular graph; distance-2 graph; spectrum; predistance polynomials.

AMS subject classifications: 05C50, 05E30.

${ }^{*}$ Research of C. Dalfó and M. A. Fiol is partially supported by AGAUR under project 2017SGR1087. Research of J. Koolen is partially supported by the National Natural Science Foundation of China under project No. 11471009, and the Chinese Academy of Sciences under its '100 talent' program.

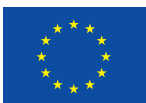

The research of C. Dalfó has also received funding from the European Union's Horizon 2020 research and innovation programme under the Marie Skłodowska-Curie grant agreement No 734922. 


\section{Preliminaries}

Let $\Gamma$ be a distance-regular graph with adjacency matrix $\boldsymbol{A}$ and $d+1$ distinct eigenvalues. The distance- $i$ graph (associated with $\Gamma$ ) is the graph $\Gamma_{i}$ having the same vertices as $\Gamma$ and in which two vertices are adjacent if and only if they are at distance $i$ in $\Gamma$. Similarly, the distance- $i$-or- $j$ graph is the graph $\Gamma_{i, j}$ with the same vertices as $\Gamma$ and in which two vertices are adjacent if and only if they are at distance $i$ or $j$ in $\Gamma$. In the recent works of Brouwer and Fiol [4, 15, it was studied the situation in which the distance- $d$ graph $\Gamma_{d}$ of $\Gamma$ (or the Kneser graph $K$ of $\Gamma$ ) with adjacency matrix $\boldsymbol{A}_{d}\left(=p_{d}(\boldsymbol{A})\right.$ ), where $p_{d}$ is the distance- $d$ polynomial, has fewer distinct eigenvalues than $\Gamma$. Examples are the so-called half antipodal ( $K$ with only one negative eigenvalue, up to multiplicity), and antipodal distance-regular graphs (where $K$ consists of disjoint copies of a complete graph).

Here we study the cases in which $\Gamma$ has few eigenvalues and its distance- 2 graph $\Gamma_{2}$ or its distance-1-or-2 graph $\Gamma_{1,2}$ are strongly regular. The main result of this paper is a characterization of such (partially) distance-regular graphs, among regular graphs with $d \in\{3,4\}$ distinct nontrivial eigenvalues, in terms of the spectrum and the mean number of vertices at maximal distance $d$ from every vertex. This can be seen as another version of the so-called spectral excess theorem. Other related characterizations of some of these cases were given by Fiol in [11, 12, 13]. For background on distance-regular graphs and strongly regular graphs, we refer the reader to Brouwer, Cohen, and Neumaier [3], Brouwer and Haemers [5], and Van Dam, Koolen and Tanaka [9].

Let $\Gamma$ be a regular (connected) graph with degree $k, n$ vertices, and spectrum $\mathrm{sp} \Gamma=$ $\left\{\theta_{0}^{m_{0}}, \theta_{1}^{m_{1}}, \ldots, \theta_{d}^{m_{d}}\right\}$, where $\theta_{0}(=k)>\theta_{1}>\cdots>\theta_{d}$, and $m_{0}=1$. In this work, we use the following scalar product on the $(d+1)$-dimensional vector space of real polynomials modulo $m(x)=\prod_{i=0}^{d}\left(x-\theta_{i}\right)$, that is, the minimal polynomial of $\boldsymbol{A}$.

$$
\langle p, q\rangle_{\Gamma}=\frac{1}{n} \operatorname{tr}(p(\boldsymbol{A}) q(\boldsymbol{A}))=\frac{1}{n} \sum_{i=0}^{d} m_{i} p\left(\theta_{i}\right) q\left(\theta_{i}\right), \quad p, q \in \mathbb{R}_{d}[x] /(m(x)) .
$$

This is a special case of the inner product of symmetric $n \times n$ real matrices $\boldsymbol{M}$ and $\boldsymbol{N}$, defined by $\langle\boldsymbol{M}, \boldsymbol{N}\rangle=\frac{1}{n} \operatorname{tr}(\boldsymbol{M} \boldsymbol{N})$. The predistance polynomials $p_{0}, p_{1}, \ldots, p_{d}$, introduced by Fiol and Garriga [17, are a sequence of orthogonal polynomials with respect to the inner product (1), normalized in such a way that $\left\|p_{i}\right\|_{\Gamma}^{2}=p_{i}(k)$ (this makes sense since it is known that $p_{i}(k)>0$ for any $i=0, \ldots, d$, see for instance Szegö [20]). As every sequence of orthogonal polynomials, the predistance polynomials satisfy a three-term recurrence of the form

$$
x p_{i}=\beta_{i-1} p_{i-1}+\alpha_{i} p_{i}+\gamma_{i+1} p_{i+1} \quad i=0,1, \ldots, d,
$$

where the constants $\beta_{i-1}, \alpha_{i}$, and $\gamma_{i+1}$ are called preintersection numbers and are the Fourier coefficients of $x p_{i}$ in terms of $p_{i-1}, p_{i}$, and $p_{i+1}$, respectively (and $\beta_{-1}=\gamma_{d+1}=0$ ), beginning with $p_{0}=1$ and $p_{1}=x$.

Some basic properties of the predistance polynomials and preintersection numbers are 
included in the following result (see Cámara, Fàbrega, Fiol, and Garriga [6], and Diego, Fàbrega, and Fiol [10]).

Lemma 1. Let $G$ be a k-regular graph with $d+1$ distinct eigenvalues and predistance polynomials $p_{0}, \ldots, p_{d}$. Given an integer $\ell \geq 0$, let $\overline{\mathcal{C}}_{\ell}$ be the average number of circuits of length $\ell$ rooted at every vertex, that is, $\overline{\mathcal{C}}_{\ell}=\frac{1}{n} \sum_{i=0}^{d} m_{i} \theta_{i}^{\ell}$. Then,

(i) $p_{0}(x)=1, p_{1}(x)=x, p_{2}(x)=\frac{1}{\gamma_{2}}\left(x^{2}-\alpha_{1} x-k\right)$.

(ii) For $i=0, \ldots, d$, the two highest terms of the predistance polynomial $p_{i}$ are given by

$$
p_{i}(x)=\frac{1}{\gamma_{1} \cdots \gamma_{i}}\left[x^{i}-\left(\alpha_{1}+\cdots+\alpha_{i-1}\right) x^{i-1}+\cdots\right] .
$$

(iii) $\alpha_{i}+\beta_{i}+\gamma_{i}=k$, for $i=0, \ldots, d$.

(iv) $\alpha_{0}=0, \beta_{0}=k, \gamma_{1}=1, \alpha_{1}=\overline{\mathcal{C}}_{3} / \overline{\mathcal{C}}_{2}$, and

$$
\gamma_{2}=\frac{\overline{\mathcal{C}}_{3}^{2}-\overline{\mathcal{C}}_{4} k+k^{3}}{k\left(\overline{\mathcal{C}}_{3}+k-k^{2}\right)} .
$$

(v) $p_{0}+p_{1}+\cdots+p_{d}=H$, where $H$ is the Hoffman polynomial [19].

(vi) For every $i=0, \ldots, d$, (any multiple of) the sum polynomial $q_{i}=p_{0}+\cdots+p_{i}$ maximizes the quotient $r\left(\theta_{0}\right) /\|r\|_{\Gamma}$ among the polynomials $r \in \mathbb{R}_{i}[x]$ (notice that $\left.q_{i}\left(\theta_{0}\right)^{2} /\left\|q_{i}\right\|_{\Gamma}^{2}=q_{i}\left(\theta_{0}\right)\right)$, and

$$
(1=) q_{0}\left(\theta_{0}\right)<q_{1}\left(\theta_{0}\right)<\cdots<q_{d}\left(\theta_{0}\right)\left(=H\left(\theta_{0}\right)=n\right) .
$$

A graph $G$ with diameter $D$ is called $m$-partially distance-regular, for some $m=$ $0, \ldots, D$, if its predistance polynomials satisfy $p_{i}(\boldsymbol{A})=\boldsymbol{A}_{i}$ for every $i \leq m$. In particular, every $m$-partially distance-regular with $m \geq 1$ must be regular (see Abiad, Van Dam, and Fiol [1]). As an alternative characterization, a graph $G$ is $m$-partially distance-regular when the intersection numbers $c_{i}(i \leq m), a_{i}(i \leq m-1), b_{i}(i \leq m-1)$ are well-defined. In this case, these intersection numbers are equal to the corresponding preintersection numbers $\gamma_{i}(i \leq m), \alpha_{i}(i \leq m-1), \beta_{i}(i \leq m-1)$, and also $k_{i}$ is well-defined and equal to $p_{i}\left(\theta_{0}\right)$ for $i \leq m$. We refer to Dalfó, Van Dam, Fiol, Garriga, and Gorissen [7] for more background.

Then, with this definition, a graph $\Gamma$ with diameter $D=d$ is distance-regular if and only it is $d$-partially distance-regular. In fact, in this case we have the following strongest proposition, which is a combination of results in Fiol, Garriga and Yebra [18], and Dalfó, Van Dam, Fiol, Garriga and Gorissen [7].

Proposition 2. A regular graph $\Gamma$ with $d+1$ different eigenvalues (and, hence, with diameter $D \leq d$ ) is distance-regular if and only if there exists a polynomial $p$ of degree $d$ such that $p(\boldsymbol{A})=\boldsymbol{A}_{d}$, in which case $p=p_{d}$. 
Lemma 3. Let $\Gamma$ be a regular graph with diameter $D$, and let $m \leq D$ be a positive integer. Let $n_{i}(u)$ be the number of vertices at distance at most $i \leq D$ from vertex $u$ in $\Gamma$, and let $\overline{n_{i}}=\frac{1}{n} \sum_{u \in V} n_{i}(u)$ be the average of these numbers of vertices for all $u \in V$. Then, for any nonzero polynomial $r \in \mathbb{R}_{i}[x]$ we have

$$
\frac{r\left(\theta_{0}\right)^{2}}{\|r\|_{\Gamma}^{2}} \leq \overline{n_{i}}
$$

with equality if and only if $r$ is a multiple of $q_{i}=p_{0}+\cdots+p_{i}$, and $q_{i}(\boldsymbol{A})=\boldsymbol{A}_{0}+\cdots+\boldsymbol{A}_{i}$.

Proof. Let $\boldsymbol{S}_{i}=\boldsymbol{A}_{0}+\cdots+\boldsymbol{A}_{i}$. As $\operatorname{deg} r \leq i$, we have $\langle r(\boldsymbol{A}), \boldsymbol{J}\rangle=\left\langle r(\boldsymbol{A}), \boldsymbol{S}_{i}\right\rangle$, where $\boldsymbol{J}$ is the all-one matrix. But $\langle r(\boldsymbol{A}), \boldsymbol{J}\rangle=\langle r, H\rangle_{\Gamma}=r\left(\theta_{0}\right)$. Then, the Cauchy-Schwarz inequality gives

$$
r^{2}\left(\theta_{0}\right) \leq\|r(\boldsymbol{A})\|^{2}\left\|\boldsymbol{S}_{i}\right\|^{2}=\|r\|_{\Gamma}^{2} \overline{n_{i}},
$$

whence (3) follows. Besides, in case of equality we have that $r$ is multiple of $q_{i}$, by Lemma 1 (vi), with $q_{i}\left(\theta_{0}\right)=\overline{n_{i}}$. Therefore, $q_{i}(\boldsymbol{A})=\alpha \boldsymbol{S}_{i}$ for some nonzero constant $\alpha$ and taking norms we conclude that $\alpha=1$.

In fact, as it was shown in Fiol [14, the above result still holds if we change the arithmetic mean of the numbers $n_{i}(u), u \in V$, by its harmonic mean.

As a consequence of Lemma 3 and Proposition 2, we have the following generalization of the spectral excess theorem, due to Fiol and Garriga [17] (for short proofs, see Van Dam [8], and Fiol, Gago and Garriga [16]).

Theorem 4. Let $\Gamma$ be a regular graph with $d+1$ distinct eigenvalues $\theta_{0}>\cdots>\theta_{d}$, and diameter $D=d$. Let $m \leq D$ be a positive integer.

(i) If $\Gamma$ is $(m-1)$-partially distance-regular for some $m<d$, and $q_{m}\left(\theta_{0}\right)=\overline{n_{m}}$, then $\Gamma$ is m-partially distance-regular.

(ii) If $q_{d-1}\left(\theta_{0}\right)=\overline{n_{d-1}}$, then $\Gamma$ is distance-regular.

\section{The case of distance-regular graphs}

Here we study the case when $\Gamma$ is a distance-regular graph with diameter three or four. In fact, in the first case everything is basically known (see Brouwer [2]), although only a combinatorial characterization was provided, whereas we think that the spectral characterization is also important. Indeed, Brouwer [2] proved the following (see also Proposition 4.2.17(i) in Brouwer, Cohen, and Neumaier [3]):

Proposition 5. [2] Let $\Gamma$ be a distance-regular graph with degree $k$ and diameter $d=3$. Then, 
(i) $\Gamma_{2}$ is strongly regular $\Longleftrightarrow c_{3}\left(a_{3}+a_{2}-a_{1}\right)=b_{1} a_{2}$.

(ii) $\Gamma_{1,2}$ is strongly regular $\Longleftrightarrow \Gamma$ has eigenvalue $-1 \Longleftrightarrow k=b_{2}+c_{3}-1$.

Notice that, in this case, $\Gamma_{1,2}$ is strongly regular if and only if its complement $\Gamma_{3}$ is. As commented in the Introduction, the last case was studied for general diameter by Brouwer and Fiol [4] and Fiol [15].

Proposition 6. Let $\Gamma$ be a distance-regular graph with diameter $D=d=3$, and eigenvalues $\theta_{0}(=k)>\theta_{1}>\theta_{2}>\theta_{3}$.

(i) The distance-2 graph $\Gamma_{2}$ is strongly regular if and only if $a_{2}-c_{3}$ is an eigenvalue of $\Gamma$.

(ii) The distance-1-or-2 graph $\Gamma_{1,2}$ is strongly regular if and only if $a_{3}-b_{2}$ is an eigenvalue of $\Gamma$.

Proof. We only prove $(i)$, as the proof of $(i i)$ is similar. As $\Gamma_{2}$ has adjacency matrix $\boldsymbol{A}_{2}=p_{2}(\boldsymbol{A})$, where $p_{2}(x)=\frac{1}{c_{2}}\left(x^{2}-a_{1} x-k\right)$, it has eigenvalues $\frac{1}{c_{2}}\left(\theta^{2}-a_{1} \theta-k\right)$, where $\theta$ is an eigenvalue of $\Gamma$. For two non-trivial eigenvalues $\eta, \theta$ of $\Gamma$, assume that $\eta^{2}-a_{1} \eta-k=$ $\theta^{2}-a_{1} \theta-k$. This implies $\theta=\eta$ or $\theta+\eta=a_{1}$. Let $\tau$ be the third non-trivial eigenvalue of $\Gamma$. Then $k+\theta+\eta+\tau=a_{1}+a_{2}+a_{3}$ and the result follows. The other direction is trivial to see.

For diameter $D=d=4$ only the case of the distance-1-or-2 graph is known (see Proposition 4.2.18 in Brouwer, Cohen, and Neumaier [3]). In the following result, we give an equivalent characterization of this case and, moreover, we study the case of the distance-2 graph which, as far as we know, it is new. The proof is as in Proposition 6. For instance, notice that, in case $(i)$, for $\Gamma_{2}$ to have only two nontrivial distinct eigenvalues, the only possibility is that $p_{2}\left(\theta_{1}\right)=p_{2}\left(\theta_{4}\right)$ and $p_{2}\left(\theta_{2}\right)=p_{2}\left(\theta_{3}\right)$.

Proposition 7. Let $\Gamma$ be a distance-regular graph with diameter four and eigenvalues $\theta_{0}(=k)>\theta_{1}>\theta_{2}>\theta_{3}>\theta_{4}$.

(i) The distance-2 graph $\Gamma_{2}$ is strongly regular if and only if $\theta_{1}+\theta_{4}=a_{1}=\theta_{2}+\theta_{3}$.

(ii) The distance-1-or-2 graph $\Gamma_{2}$ is strongly regular if and only if $\theta_{1}+\theta_{4}=a_{1}-c_{2}=$ $\theta_{2}+\theta_{3}$.

An example of distance-regular graph satisfying the conditions of Proposition 7 is the Hamming graph $H(4,3)$ (see Example 2 in the next section). Another example would be the (possible) graph corresponding to the feasible array $\{39,32,20,2 ; 1,4,16,30\}$ (see Brouwer, Cohen, and Neumaier [3, p. 420]). If it exists, this would be a graph with $n=768$ vertices and spectrum $39^{1}, 15^{52}, 7^{117},-1^{468},-9^{130}$. In this case, its distance- 2 graph would have spectrum $312^{1}, 24^{182},-8^{585}$. 


\section{The case of regular graphs}

Now we want to conclude the same result as above but only requiring that the graph $\Gamma$ is regular. In this case, we use the predistance polynomials and preintersection numbers. Notice that now $p_{i}(\boldsymbol{A})$ is not necessarily the distance- $i$ matrix $\boldsymbol{A}_{i}$ (usually not even a 0-1 matrix). However, as above, we consider that $p_{2}(\boldsymbol{A})$ has only three distinct eigenvalues.

\subsection{The case of diameter three}

We begin with the case of $d=3$ (that is, assuming that $\Gamma$ has four distinct eigenvalues).

Theorem 8. Let $\Gamma$ be a regular graph with degree $k, n$ vertices, spectrum $\operatorname{sp} \Gamma=\left\{\theta_{0}, \theta_{1}^{m_{1}}\right.$, $\left.\underline{\theta}_{2}^{m_{2}}, \theta_{3}^{m_{3}}\right\}$, where $\theta_{0}(=k)>\theta_{1}>\theta_{2}>\theta_{3}$, and preintersection number $\gamma_{2}$ given by (2). Let $\overline{k_{3}}=\frac{1}{n} \sum_{u \in V} k_{3}(u)$ be the average number of vertices at distance 3 from every vertex in $\Gamma$. Consider the polynomials

$$
\begin{aligned}
& s_{1}(x)=x^{2}-\left(\theta_{1}+\theta_{3}-\gamma_{2}\right) x+\gamma_{2}+\theta_{2}\left(\theta_{1}-\theta_{2}+\theta_{3}\right), \\
& s_{2}(x)=x^{2}-\left(\theta_{1}+\theta_{2}-\gamma_{2}\right) x+\gamma_{2}+\theta_{3}\left(\theta_{1}-\theta_{3}+\theta_{2}\right) .
\end{aligned}
$$

Then,

$$
\overline{k_{3}} \leq \frac{n \sum_{i=1}^{3} m_{i}\left(s_{j}\left(\theta_{i}\right)-\tau_{j}\right)^{2}}{\sum_{i=0}^{3} m_{i}\left(s_{j}\left(\theta_{i}\right)-\tau_{j}\right)^{2}}, \quad j=1,2,
$$

where

$$
\tau_{j}=\frac{s_{j}\left(\theta_{0}\right) \sum_{i=1}^{3} m_{i} s_{j}\left(\theta_{i}\right)-\sum_{i=1}^{3} m_{i} s_{j}\left(\theta_{i}\right)^{2}}{s_{j}\left(\theta_{0}\right)(n-1)-\sum_{i=1}^{3} m_{i} s_{j}\left(\theta_{i}\right)}, \quad j=1,2 .
$$

Equality in (6) holds for some $j \in\{1,2\}$ if and only if $\Gamma$ is a distance-regular graph and its distance-2 graph $\Gamma_{2}$ is strongly regular, with eigenvalues

$$
\lambda_{0}=n-\overline{k_{3}}-\theta_{0}-1, \quad \lambda_{1}=\left(\left(\theta_{1}-\theta_{2}\right)\left(\theta_{2}-\theta_{3}\right)-\tau_{1}\right) / \gamma_{2}, \text { and } \lambda_{2}=-\tau_{1} / \gamma_{2},
$$

or

$$
\lambda_{0}=n-\overline{k_{3}}-\theta_{0}-1, \quad \lambda_{1}=-\tau_{2} / \gamma_{2}, \text { and } \lambda_{2}=\left(\left(\theta_{1}-\theta_{3}\right)\left(\theta_{3}-\theta_{2}\right)-\tau_{2}\right) / \gamma_{2},
$$

where $k_{3}=\overline{k_{3}}$ is the constant value of the number of vertices at distance 3 from any vertex in $\Gamma$.

Proof. Taking into account that the eigenvalues of $p_{2}(\boldsymbol{A})$ interlace those of $\Gamma$ (because of the orthogonality of the predistance polynomials with respect to the scalar product in (1), see for instance Cámara, Fàbrega, Fiol, and Garriga [6], or Szegö [20]), the only possible cases are:

1. $p_{2}\left(\theta_{1}\right)=p_{2}\left(\theta_{3}\right)=\sigma_{1} / \gamma_{2}$ and $p_{2}\left(\theta_{2}\right)=-\tau_{1} / \gamma_{2}$, 
2. $p_{2}\left(\theta_{1}\right)=p_{2}\left(\theta_{2}\right)=\sigma_{2} / \gamma_{2}$ and $p_{2}\left(\theta_{3}\right)=-\tau_{2} / \gamma_{2}$,

where $\sigma_{j}$ and $\tau_{j}$, for $j=1,2$, are constants. We only prove the first case, as the other is similar. The main idea is to apply Lemma 3 with a polynomial $r \in \mathbb{R}_{2}[x]$ having the desired properties of (any multiple of) $q_{2}$. To this end, let us assume that $p_{2}\left(\theta_{1}\right)=p_{2}\left(\theta_{3}\right)=\sigma_{1} / \gamma_{2}$, and $p_{2}\left(\theta_{2}\right)=-\tau_{1} / \gamma_{2}$ where $\sigma_{1}$ and $\tau_{1}$ are constants. Thus, if we consider a generic monic polynomial $r(x)=x^{2}+\alpha x+\beta=\gamma_{2} q_{2}(x)$, where $q_{2}(x)=p_{2}(x)+x+1$, we must have

$$
\begin{aligned}
& r\left(\theta_{1}\right)=\theta_{1}^{2}+\alpha \theta_{1}+\beta=\sigma_{1}+\gamma_{2} \theta_{1}+\gamma_{2}, \\
& r\left(\theta_{2}\right)=\theta_{2}^{2}+\alpha \theta_{2}+\beta=-\tau_{1}+\gamma_{2} \theta_{2}+\gamma_{2}, \\
& r\left(\theta_{3}\right)=\theta_{3}^{2}+\alpha \theta_{3}+\beta=\sigma_{1}+\gamma_{2} \theta_{3}+\gamma_{2} .
\end{aligned}
$$

From the first and last equation we get $\alpha=\gamma_{2}-\theta_{1}-\theta_{3}$ and, hence, the second equation yields $\beta=\gamma_{2}+\theta_{2}\left(\theta_{1}-\theta_{2}+\theta_{3}\right)-\tau_{1}$. Then, we must take $r(x)=s_{1}(x)-\tau_{1}$, where $s_{1}(x)$ is as in (4), and (3) yields

$$
\Phi\left(\tau_{1}\right)=\frac{r\left(\theta_{0}\right)^{2}}{\|r\|_{\Gamma}^{2}}=\frac{n\left(s\left(\theta_{0}\right)-\tau_{1}\right)^{2}}{\sum_{i=0}^{3} m_{i}\left(s\left(\theta_{i}\right)-\tau_{1}\right)^{2}} \leq \overline{s_{2}}=n-\overline{k_{3}} .
$$

Now, to have the best result in (8), and, since we are mostly interested in the case of equality, we find the maximum of the function $\Phi$, which is attained at $\tau_{1}$ given by (7). Then, as $\overline{s_{2}}=n-\overline{k_{3}}$, the claimed inequality follows. Moreover, in case of equality, we know, by Theorem 4, that $\Gamma$ is distance-regular with $r(x)=\gamma q_{d-1}(x)$ for some constant $\gamma$, which it is $\gamma=\gamma_{2}$. Then, we get (with standard notation $P_{i j}=p_{j}\left(\theta_{i}\right)$ )

$$
\begin{aligned}
& P_{22}=p_{2}\left(\theta_{2}\right)=-\frac{\tau_{1}}{\gamma_{2}}, \\
& P_{i 2}=p_{2}\left(\theta_{i}\right)=\frac{\sigma_{1}}{\gamma_{2}}=\frac{1}{\gamma 2}\left(\left(\theta_{1}-\theta_{2}\right)\left(\theta_{2}-\theta_{3}\right)-\tau_{1}\right), \quad i=1,3 .
\end{aligned}
$$

To prove the converse, we only need to carry out a simple computation. Indeed, assume that $\Gamma$ is a distance-regular graph, with $k_{i}$ being the vertices at distance $i=1,2,3$ from any vertex $\left(k_{1}=k\right)$, and $p_{2}\left(\theta_{1}\right)=p_{d}\left(\theta_{3}\right)$. Then, the same reasoning as in Proposition 6 gives $a_{1}=\theta_{1}+\theta_{3}$. Then, from $k b_{1}=c_{2} k_{2}=c_{2}\left(n-k_{3}-k-1\right)$ and $a_{1}+b_{1}+1=k$, we get that $c_{2}=\frac{k\left(k-1-\theta_{1}-\theta_{3}\right)}{n-k_{3}-k-1}$. Thus, by putting $\gamma_{2}=c_{2}$ in $s_{1}(x)$ of (4) to compute $\tau_{1}$ in (7), the inequality (6) becomes an equality ( since $\overline{k_{3}}=k_{3}$ ).

The following result gives similar conditions for $\Gamma$ to be distance-regular with the distance-1-or-2 graph $\Gamma_{1,2}$ being strongly regular.

Theorem 9. Let $\Gamma$ be a regular graph with degree $k, n$ vertices, spectrum $\operatorname{sp} \Gamma=\left\{\theta_{0}, \theta_{1}^{m_{1}}\right.$, $\left.\theta_{2}^{m_{2}}, \theta_{3}^{m_{3}}\right\}$, where $\theta_{0}(=k)>\theta_{1}>\theta_{2}>\theta_{3}$, and preintersection number $\gamma_{2}$. Let $\overline{k_{3}}=$ 
$\frac{1}{n} \sum_{u \in V} k_{3}(u)$ be the average number of vertices at distance 3 from every vertex in $\Gamma$. Consider the polynomials

$$
\begin{aligned}
& s_{1}(x)=x^{2}-\left(\theta_{1}+\theta_{3}\right) x+\gamma_{2}+\theta_{2}\left(\theta_{1}-\theta_{2}+\theta_{3}\right), \\
& s_{2}(x)=x^{2}-\left(\theta_{1}+\theta_{2}\right) x+\gamma_{2}+\theta_{3}\left(\theta_{1}-\theta_{3}+\theta_{2}\right) .
\end{aligned}
$$

Then,

$$
\overline{k_{3}} \leq \frac{n \sum_{i=1}^{3} m_{i}\left(s_{j}\left(\theta_{i}\right)-\tau_{j}\right)^{2}}{\sum_{i=0}^{3} m_{i}\left(s_{j}\left(\theta_{i}\right)-\tau_{j}\right)^{2}}, \quad j=1,2,
$$

where

$$
\tau_{j}=\frac{s_{j}\left(\theta_{0}\right) \sum_{i=1}^{3} m_{i} s_{j}\left(\theta_{i}\right)-\sum_{i=1}^{3} m_{i} s_{j}\left(\theta_{i}\right)^{2}}{s_{j}\left(\theta_{0}\right)(n-1)-\sum_{i=1}^{3} m_{i} s_{j}\left(\theta_{i}\right)}, \quad j=1,2 .
$$

Equality in (11) holds with some $j \in\{1,2\}$ if and only if $\Gamma$ is a distance-regular graph and its distance-1-or-2 graph $\Gamma_{1,2}$ is strongly regular,

Example 1. The Odd graph $\mathrm{O}(4)$ with 7 points, has $n=\left(\begin{array}{l}7 \\ 3\end{array}\right)=35=1+4+12+18$ vertices, diameter $d=3$, intersection array $\{4,3,3 ; 1,1,2\}$, and spectrum $4^{1}, 2^{14},-1^{14},-3^{6}$. Then, the functions $\Phi\left(\tau_{j}\right)$ in (6) with $j=1,2$ have maximum values at $\tau_{1}=18 / 5$ and $\tau_{2}=-8$, respectively, and their values are $\Phi(18 / 5)=138 / 7$ and $\Phi(-8)=22$. Then, since both numbers are greater than $k_{3}=18$, its distance- 2 graph $\Gamma_{2}$ is not strongly regular.

On the other hand the function $\Phi\left(\tau_{1}\right)$ in (11) has maximum value at $\tau_{1}=4$, and $\Phi(4)=18=k_{3}$. Hence, its distance-1-or-2 graph $\Gamma_{1,2}$ (and, hence, also $\Gamma_{3}$ ) is strongly regular with $p_{1}(x)+p_{2}(x)=x^{2}+x-4$, and spectrum $16^{1}, 2^{20},-4^{14}$.

\subsection{The case of diameter four}

The following result deals with the case of $d=4$. As in the case of Theorem 9, we omit this proof as goes along the same lines of reasoning as in Theorem 8 .

Theorem 10. Let $\Gamma$ be a regular graph with degree $k, n$ vertices, spectrum $\operatorname{sp} \Gamma=\left\{\theta_{0}, \theta_{1}^{m_{1}}\right.$, $\left.\theta_{2}^{m_{2}}, \theta_{3}^{m_{3}}, \theta_{4}^{m_{4}}\right\}$, where $\theta_{0}(=k)>\theta_{1}>\theta_{2}>\theta_{3}>\theta_{4}$, such that $\theta_{1}+\theta_{4}=\theta_{2}+\theta_{3}$, and preintersection number $\gamma_{2}$. Let $\overline{n_{2}}=\frac{1}{n} \sum_{u \in V} n_{2}(u)$ be the average number $n_{2}(u)=\left|N_{2}(u)\right|$ of vertices at distance at most 2 from every vertex $u$ in $\Gamma$. Consider the polynomials

$$
\begin{aligned}
& s_{1}(x)=x^{2}-\left(\theta_{2}+\theta_{3}-\gamma_{2}\right) x+\theta_{2} \theta_{3}, \\
& s_{2}(x)=x^{2}-\left(\theta_{2}+\theta_{3}\right) x+\gamma_{2}-\theta_{2} \theta_{3} .
\end{aligned}
$$

Then,

$$
\overline{n_{2}} \geq \Phi\left(\tau_{j}\right)=\frac{n\left(s_{j}\left(\theta_{0}\right)-\tau_{j}\right)^{2}}{\sum_{i=0}^{4} m_{i}\left(s_{j}\left(\theta_{i}\right)-\tau_{j}\right)^{2}}, \quad j=1,2,
$$

where

$$
\tau_{j}=\frac{s_{j}\left(\theta_{0}\right) \sum_{i=1}^{4} m_{i} s_{j}\left(\theta_{i}\right)-\sum_{i=1}^{4} m_{i} s_{j}\left(\theta_{i}\right)^{2}}{s_{j}\left(\theta_{0}\right)(n-1)-\sum_{i=1}^{4} m_{i} s_{j}\left(\theta_{i}\right)}, \quad j=1,2
$$


Equality in (13) holds with $j=1$ or $j=2$ if and only if $\Gamma$ is a 2-partially distance-regular graph and its distance-2 or distance-1-or-2 graph, respectively, is strongly regular.

Example 2. The Hamming graph $H(4,3)$, with $n=3^{4}=81$ vertices and diameter $d=4$, has intersection array $\{8,6,4,2 ; 1,2,3,4\}$, so that $k_{4}=16$, and spectrum $8^{1}, 5^{8}, 2^{24},-1^{32},-4^{16}$. Then, the function $\Phi\left(\tau_{j}\right)$ in 13 with $j=1$ has a maximum at $\tau_{1}=4$, and its value is $\Phi(4)=33=s_{2}$. Then, $P_{14}=P_{44}$ and $P_{24}=P_{34}$. Indeed, its distance- 2 polynomial is $p_{2}(x)=\frac{1}{2}\left(x^{2}-x-8\right)$ with values $p_{4}(8)=24, p_{4}(5)=6$, $p_{4}(2)=-3, p_{4}(-1)=-3$, and $p_{4}(-4)=6$. Hence, the distance-2 graph $\Gamma_{2}$ is strongly regular with spectrum $24^{1}, 6^{24},-3^{56}$.

Example 3. The Odd graph $\mathrm{O}(5)$ with 9 points, has $n=\left(\begin{array}{l}9 \\ 4\end{array}\right)=126=1+5+20+$ $40+60$ vertices, diameter $d=4$, intersection array $\{5,4,4,3 ; 1,1,2,2\}$, and spectrum $5^{1}, 3^{27}, 1^{42},-2^{48},-4^{8}$. Then, the function $\Phi\left(\tau_{j}\right)$ in 13 with $j=2$ has a maximum at $\tau_{2}=3$, and its value is $\Phi(4)=26=s_{2}$. Then, its distance-1-or-2 polynomial is $p_{1,2}(x)=p_{1}(x)+p_{2}(x)=x^{2}+x-5$ with values $p_{1,2}(5)=25, p_{1,2}(3)=7, p_{1,2}(1)=-3$, $p_{1,2}(-2)=-3$, and $p_{1,2}(-4)=7$. Hence, the distance-1-or-2 graph $\Gamma_{1,2}$ is strongly regular with spectrum $25^{1}, 7^{35},-3^{90}$.

\section{References}

[1] A. Abiad, E. R. van Dam, and M. A. Fiol, Some spectral and quasi-spectral characterizations of distance-regular graphs, J. Combin. Theory, Ser. A 143 (2016) 1-18.

[2] A. E. Brouwer, Distance regular graphs of diameter 3 and strongly regular graphs, Discrete Math. 49 (1984) 101-103.

[3] A. E. Brouwer, A. M. Cohen, and A. Neumaier, Distance-Regular Graphs, SpringerVerlag, Berlin-New York, 1989.

[4] A. E. Brouwer and M. A. Fiol, Distance-regular graphs where the distance-d graph has fewer distinct eigenvalues, Linear Algebra Appl. 480 (2015) 115-126.

[5] A. E. Brouwer and W. H. Haemers, Spectra of Graphs, Springer, 2012; available online at http://homepages.cwi.nl/ aeb/math/ipm/.

[6] M. Cámara, J. Fàbrega, M. A. Fiol, and E. Garriga, Some families of orthogonal polynomials of a discrete variable and their applications to graphs and codes, Electron. J. Combin. 16(1) (2009) \#R83.

[7] C. Dalfó, E. R. van Dam, M. A. Fiol, E. Garriga, and B. L. Gorissen, On almost distance-regular graphs, J. Combin. Theory, Ser. A 118 (2011) 1094-1113.

[8] E. R. van Dam, The spectral excess theorem for distance-regular graphs: a global (over)view, Electron. J. Combin. 15(1) (2008) \#R129. 
[9] E. R. van Dam, J. H. Koolen, and H. Tanaka, Distance-regular graphs, Electron. J. Combin. (2016) \#DS22.

[10] V. Diego, J. Fàbrega, and M. A. Fiol, Equivalent characterizations of the spectra of graphs and applications to measures of distance-regularity, submitted (2016).

[11] M. A. Fiol, An eigenvalue characterization of antipodal distance-regular graphs, Electron. J. Combin. 4 (1997) \#R30.

[12] M. A. Fiol, A quasi-spectral characterization of strongly distance-regular graphs, Electron. J. Combin. 7 (2000) \#R51.

[13] M. A. Fiol, Some spectral characterization of strongly distance-regular graphs, Combin. Probab. Comput. 10 (2001), no. 2, 127-135.

[14] M. A. Fiol, Algebraic characterizations of distance-regular graphs, Discrete Math. 246 (2002) 111-129.

[15] M. A. Fiol, The spectral excess theorem for distance-regular graphs having distance- $d$ graph with fewer distinct eigenvalues, J. Algebraic Combin. 43 (2016), no. 4, 827-836.

[16] M. A. Fiol, S. Gago, and E. Garriga, A simple proof of the spectral excess theorem for distance-regular graphs, Linear Algebra Appl. 432 (2010) 2418-2422.

[17] M. A. Fiol and E. Garriga, From local adjacency polynomials to locally pseudodistance-regular graphs, J. Combin. Theory Ser. B 71 (1997) 162-183.

[18] M. A. Fiol, E. Garriga, and J. L. A. Yebra, Locally pseudo-distance-regular graphs, J. Combin. Theory Ser. B 68 (1996) 179-205.

[19] A. J. Hoffman, On the polynomial of a graph, Amer. Math. Monthly 70 (1963) 30-36.

[20] G. Szegö, Orthogonal Polynomials, 4th edition, American Mathematical Society, Providence, R.I., 1975. 